Beteiligung - ein Programm für Politik,
Wirtschaft und Gesellschaft

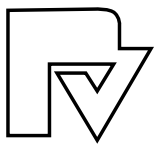


Hans-Peter Meister · Felix Oldenburg

\section{Beteiligung - \\ ein Programm für Politik, Wirtschaft und Gesellschaft}

Physica-Verlag

Ein Unternehmen von Springer 


\section{Dr. Hans-Peter Meister \\ IFOK GmbH}

Berliner Ring 89

64625 Bensheim

hans-peter.meister@ifok.de

www.ifok.de
Felix Oldenburg, M.A., M.P.M.

IFOK GmbH

Reinhardtstraße 58

10117 Berlin

felix.oldenburg@ifok.de

ISBN 978-3-7908-1601-3

e-ISBN 978-3-7908-1935-9

DOI 10.1007/978-3-7908-1935-9

Bibliografische Information der Deutschen Nationalbibliothek

Die Deutsche Nationalbibliothek verzeichnet diese Publikation in der Deutschen Nationalbibliografie; detaillierte bibliografische Daten sind im Internet über http://dnb.d-nb.de abrufbar.

(c) 2008 Physica-Verlag Heidelberg

Dieses Werk ist urheberrechtlich geschützt. Die dadurch begründeten Rechte, insbesondere die der Übersetzung, des Nachdrucks, des Vortrags, der Entnahme von Abbildungen und Tabellen, der Funksendung, der Mikroverfilmung oder der Vervielfältigung auf anderen Wegen und der Speicherung in Datenverarbeitungsanlagen, bleiben, auch bei nur auszugsweiser Verwertung, vorbehalten. Eine Vervielfältigung dieses Werkes oder von Teilen dieses Werkes ist auch im Einzelfall nur in den Grenzen der gesetzlichen Bestimmungen des Urheberrechtsgesetzes der Bundesrepublik Deutschland vom 9. September 1965 in der jeweils geltenden Fassung zulässig. Sie ist grundsätzlich vergütungspflichtig. Zuwiderhandlungen unterliegen den Strafbestimmungen des Urheberrechtsgesetzes.

Die Wiedergabe von Gebrauchsnamen, Handelsnamen, Warenbezeichnungen usw. in diesem Werk berechtigt auch ohne besondere Kennzeichnung nicht zu der Annahme, dass solche Namen im Sinne der Warenzeichen- und Markenschutz-Gesetzgebung als frei zu betrachten wären und daher von jedermann benutzt werden dürften.

Herstellung: LE-TEX Jelonek, Schmidt \& Vöckler GbR, Leipzig

Einbandgestaltung: WMX Design $\mathrm{GmbH}$, Heidelberg

Gedruckt auf säurefreiem Papier

987654321

springer.com 
Während der Arbeit an diesem Buch haben wir vom Tod unseres Freundes, Mentors und Beirats Dr. Frank Niethammer erfahren. Er war eine starke Führungspersönlichkeit mit klaren Überzeugungen. Durch seinen Respekt vor Menschen mit anderen Meinungen und seine Offenheit zum Gespräch mit ihnen hat er uns gelehrt, wie durch Dialog Blockaden überwunden werden können. Seinem Andenken widmen wir dieses Buch. 


\section{Inhalt}

Einleitung 1

Die Wiederentdeckung der Beteiligung:

Wie Gesellschaft gestaltet werden kann 5

Konflikte bewältigen:

Politische Mediation gegen erstarrte Rituale

Mehrsektorale Partnerschaft:

Umsetzen, was ich nicht verordnen kann

Diskursive Politikgestaltung:

Besser entscheiden

Exkurs: Wie man künstlich Relevanz erzeugt

Vom Stakeholdermanagement zu CSR:

Glaubwürdig kommunizieren

Foresight und Innovation:

Zukunft denken - Heute gestalten

Modern regieren:

Neue Wege der Bürgerbeteiligung

Danksagung

Über die Autoren 